\title{
Avaliação de Jogos Educacionais Multiusuários: Uma Revisão Sistemática da Literatura
}

\author{
Daniela C. C. Peixoto ${ }^{1}$, Silas T. Campos ${ }^{1}$, Rodolfo F. Resende ${ }^{2}$ \\ ${ }^{1}$ Departamento de Computação - Centro Federal de Educação Tecnológica de Minas \\ Gerais (CEFET-MG) - Belo Horizonte - MG - Brasil \\ ${ }^{2}$ Departamento de Ciência da Computação - Universidade Federal de Minas Gerais \\ (UFMG) - Belo Horizonte - MG - Brasil \\ cascini@decom.cefetmg.br,silastolentino@gmail.com, rodolfo@dcc.ufmg.br
}

\begin{abstract}
The increasing interest in educational games leads to the problem of how to assess these games in order to measure their real benefits. This paper presents a Systematic Literature Review that shows the assessments employed into multiplayer games for educational purposes. Few attempts were proposed in order to support practitioners evaluating educational games. Usually the methods cover few characteristics of the game environment, varying according to the needs and experiences of its designer. Based on this literature review, we selected a set of questions to guide the game evaluation.
\end{abstract}

Resumo. O crescente interesse em jogos educacionais gera a necessidade de utilizar métodos de avaliação que possam medir o real beneficio desses jogos. Este trabalho apresenta uma Revisão Sistemática da Literatura que analisa os métodos de avaliação dos jogos educacionais multiusuários. Algumas tentativas de avaliação foram propostas com o objetivo de apoiar os projetistas e usuários desses jogos. Usualmente, esses métodos cobrem algumas características do ambiente de jogo, variando de acordo com as necessidades e experiências do projetista. Com base na revisão da literatura, nós consolidamos um conjunto de questões com o objetivo de guiar a avaliação de um jogo.

\section{Introdução}

Um dos grandes desafios enfrentados pela sociedade e em particular pelas universidades é a necessidade de tornar o processo de formação e treinamento das pessoas mais efetivo e eficaz. A expectativa de uma maior qualidade na formação e treinamento não tem sido satisfeita de uma maneira geral e existe um aumento da insatisfação da indústria em relação à preparação dos estudantes de vários setores [Callahan e Pedigo 2002, Exter 2014]. Soma-se a isso a dificuldade de aplicação prática dos conceitos apresentados em sala de aula com a metodologia de ensino tradicional, centrada no professor [Chen et al. 2008]. Uma forma de melhorar este cenário consiste na utilização de tecnologias e métodos de apoio ao ensino como, por exemplo, desenvolvimento de atividades em projetos no contexto da indústria de software, realização de programas de residência, jogos e simuladores. 
Mudanças profundas no processo de educação e nas instituições de ensino são esperadas com a utilização de novas tecnologias educacionais e pedagógicas [Martin et al. 2011]. Atualmente, é possível notar adaptações da metodologia tradicional. Cursos face-a-face, centrados no professor, que são comumente oferecidos por universidades do mundo inteiro, estão sendo substituídos por cursos mais dinâmicos, híbridos com a utilização de tecnologias inovadoras. Exemplos importantes incluem os cursos on-line e gratuitos (MOOC - Massive Open Online Course) oferecidos por universidades renomadas que operam numa escala global. Outros exemplos incluem a utilização de jogos de simulação como método complementar ao ensino de determinados assuntos abordados em disciplinas de graduação como, por exemplo, SimSE [Navarro 2006] e SPIAL [Peixoto et al. 2014].

A utilização de jogos educacionais é uma das formas de mudança de paradigma de ensino: do aprendizado ao se escutar (learning by listen) para o aprendizado ao se fazer (learning by doing) [Garris 2002]. Uma razão para a sua adoção é que existem evidências empíricas que indicam que os jogos educacionais são ferramentas efetivas para a melhoria do aprendizado e compreensão de assuntos complexos [Peixoto et al. 2014]. Os jogos educacionais permitem aumentar o interesse dos estudantes nos assuntos abordados em sala de aula. Além disso, possibilitam que o professor aborde certos aspectos que não são satisfatoriamente cobertos nas atividades práticas da disciplina. A variedade de jogos educacionais disponíveis na literatura [Pietruchinski et al. 2011, Peixoto et al. 2014] mostra que esta é uma área de pesquisa atrativa e com grandes possibilidades de expansão.

Apesar do potencial dos jogos educacionais e o crescente interesse, a adoção desses jogos em um contexto educacional formal permanece limitado [De Grove et al. 2012]. Uma das causas é a falta de avaliações concretas e confiáveis. Observa-se que muitas das decisões sobre a utilização de jogos educacionais são baseadas em suposições sobre a capacidade desses jogos de auxiliar no aprendizado de maneira efetiva e eficiente [Hays 2005].

Dentre as diversas dimensões, os jogos podem ser classificados em jogos para um jogador ou monousuários, em que a execução prevê um único usuário como jogador, e os jogos para múltiplos usuários ou multiusuários, em que a execução prevê mais de um usuário como participante do tema desenvolvido no jogo. Neste contexto, realizamos uma revisão sistemática da literatura para identificar os métodos e técnicas utilizadas na avaliação do uso de jogos educacionais multiusuários. A utilização desses jogos, tanto aqueles com foco educacional quanto os de entretenimento, apresenta uma grande expansão no cenário mundial. Os jogos multiusuários permitem a exploração de aspectos que não poderiam ser cobertos em jogos com um único usuário como, por exemplo, a colaboração, a sociabilidade e a competição.

O restante deste trabalho está estruturado da seguinte forma. A Seção 2 apresenta os trabalhos relacionados. A Seção 3 apresenta a metodologia de pesquisa e os resultados obtidos. A Seção 4 apresenta as considerações finais e trabalhos futuros. 


\section{Trabalhos Relacionados}

Em seu trabalho, Mayer (2012) propõe uma metodologia genérica para avaliação de jogos educacionais ${ }^{1}$. Para elaborar o modelo de avaliação, foram realizadas várias baterias de testes com 12 jogos diferentes. Para isso, a pesquisa contou com 2.164 usuários colaboradores entre 17 e 75 anos. Os dados foram coletados antes, durante e depois dos jogos. Como resultados, os autores definiram um modelo conceitual e um método para a sua operacionalização, ou seja, para a aplicação prática no contexto de jogos educacionais. O modelo é baseado em questões que devem ser avaliadas antes, durante e depois dos jogos como, por exemplo, satisfação e desempenho.

O trabalho de Villalta et al. (2011) apresenta um guia com diretrizes para o desenvolvimento de jogos educacionais multiusuários. Para elaborar este guia os autores realizaram as seguintes atividades: (1) avaliaram a implementação de um jogo e identificaram os seus principais problemas; (2) definiram um conjunto de diretrizes que foram divididas em seis dimensões: mecânica do jogo, progressão do jogo, metodologia, colaboração, informações na tela e experiência holística; (3) redefiniram o jogo com base nessas diretrizes; e (4) realizaram um estudo de caso com 20 estudantes entre $11 \mathrm{e}$ 13 anos em uma escola no Chile. Os autores observaram que o guia mostrou-se eficaz na melhoria do aprendizado dos alunos, evitando a ocorrência dos problemas anteriormente identificados. Isso sugere a importância de se utilizar guias para a criação e avaliação de jogos educacionais.

Keller (2010) desenvolveu o modelo ARCS (Attention, Relevance, Confidence, Satisfaction) que tem por objetivo empregar estratégias motivacionais no projeto de materiais educacionais. Esse modelo pode ser utilizado para a avaliação da motivação dos estudantes ao utilizarem materiais educacionais, como, por exemplo, os jogos educacionais.

Savi (2011) propõe um modelo para a avaliação da qualidade de jogos educacionais. Savi apresenta toda a estrutura teórica do seu modelo, incluindo um questionário para a coleta dos dados. O modelo foi testado em três jogos educacionais manuais, sendo dois de tabuleiro e um terceiro que utiliza cartas, papel e caneta. Segundo o autor, os primeiros resultados da aplicação do método de avaliação foram satisfatórios.

\section{Metodologia de Pesquisa}

Para realização deste trabalho utilizamos o método de Revisão Sistemática da Literatura (Systematic Literature Review - SLR) [Kitchenham e Charters 2007] que é composto, basicamente, de três fases: planejamento, condução e documentação dos resultados. Nesta seção, apresentamos o protocolo que foi produzido durante a fase de planejamento. Para a elaboração deste protocolo foram realizadas as atividades que estão descritas nas seções seguintes, juntamente com os resultados obtidos.

\subsection{Especificação da questão de pesquisa}

A definição da questão de pesquisa (QP) é uma atividade crucial que norteia todo o processo da revisão: (i) no processo de busca são definidos os termos de pesquisa

\footnotetext{
${ }^{1}$ Neste trabalho utilizamos o termo "jogos educacionais" para nos referirmos a "jogos sérios".
} 
(strings) de acordo com o conteúdo abordado pela QP; (ii) no processo de extração de dados são coletados os dados relacionados a essa QP; e (iii) no processo de análise de dados são sintetizados os dados de modo que a questão possa ser respondida.

Com base no objetivo principal deste trabalho que é identificar os métodos de avaliação do uso de jogos educacionais, mais especificamente, em jogos multiusuários, definimos a seguinte questão de pesquisa:

\section{QP - Como os jogos educacionais multiusuários são avaliados atualmente?}

O objetivo desta questão é identificar os pontos fortes e fracos dos métodos de avaliação utilizados atualmente. Essa QP foi refinada nas seguintes questões:

QP 1.1- Quais métodos de avaliação são utilizados?

QP 1.2- Quais dimensões são avaliadas?

QP 1.3- Quão satisfatórios têm sido os resultados das avaliações?

\subsection{Definição da busca}

Os estudos primários, ou seja, os artigos selecionados para a análise foram obtidos das seguintes bases de dados: ACM Digital Library, IEEEXplore, e Elsevier Science Direct.

Para realizar a pesquisa, definimos uma string de busca (SB) utilizando o critério PICOC [Kitchenham e Charters 2007]:

\section{(educational OR education OR serious) AND (game OR simulation) AND (multiplayer OR multiplayer OR "multi player")}

As palavras chaves na SB foram definidas em inglês com o objetivo de identificar um número mais expressivo de estudos primários relacionados à QP.

\subsection{Seleção dos estudos}

A busca foi realizada no período de 10 anos, 2004 a 2014. A Tabela 1 sumariza esta busca.

Tabela 1 - Quantidade de resultados encontrados por biblioteca.

\begin{tabular}{|c|c|c|}
\hline Biblioteca & Resultados & Porcentagem \\
\hline ACM Digital Library & 113 & $32 \%$ \\
\hline IEEEXplore & 136 & $38 \%$ \\
\hline ScienceDirect & 106 & $30 \%$ \\
\hline Total & $\mathbf{3 5 5}$ & $\mathbf{1 0 0} \%$ \\
\hline
\end{tabular}

Para selecionar os estudos, foram definidos os critérios de inclusão e exclusão, com base na QP. Esses critérios foram criados de maneira a serem interpretados de forma não ambígua e, assim, permitir a seleção adequada dos estudos. A Tabela 2 apresenta os critérios de inclusão e exclusão.

Tendo como base o guia de Kitchenham e Charters (2007), executamos os seguintes passos para obter os estudos primários:

1. Análise de títulos: Para eliminar estudos duplicados e irrelevantes, analisamos somente os títulos dos 355 artigos encontrados. Após essa etapa foram eliminados 127 artigos, restando 228 artigos. 
2. Análise do resumo: Para eliminar os estudos que não estavam relacionados com as QPs, analisamos os resumos dos 228 artigos. Após essa etapa foram eliminados outros 153 artigos, restando 75 artigos.

3. Análise rápida do texto: Para eliminar os estudos que não atendiam aos critérios de inclusão, realizamos uma leitura rápida (scanning) dos 75 artigos. Após essa etapa, eliminamos mais 53 artigos, restando 22 artigos.

4. Leitura detalhada: Analisamos detalhadamente os 22 artigos restantes e excluímos os trabalhos duplicados e os que se enquadravam em um dos critérios de exclusão. Desta forma, excluímos mais 5 artigos, restando 17 artigos.

Tabela 2 - Critérios de inclusão e exclusão.

\begin{tabular}{|c|c|}
\hline Critério & Descrição \\
\hline Inclusão & $\begin{array}{l}\text { Todos os artigos que desenvolvem, comentam ou analisam o } \\
\text { processo de avaliação de um jogo educacional multiusuário. }\end{array}$ \\
\hline Exclusão & $\begin{array}{l}\text { Artigos resumidos, resumos ou apresentações. } \\
\text { Artigos que não estão em inglês. } \\
\text { Artigos duplicados ou que falam sobre o mesmo jogo. Neste caso, } \\
\text { incluir o artigo mais completo. } \\
\text { Artigos que descrevem jogos monousuários. } \\
\text { Artigos que descrevem jogos sem foco no ensino. } \\
\text { Artigos que avaliam aspectos que saem do escopo do ensino (ex.: } \\
\text { análise psicológica, gênero dos jogadores). } \\
\text { Artigos que não descrevem um jogo específico. } \\
\text { Artigos que não descrevem a avaliacão do jogo. }\end{array}$ \\
\hline
\end{tabular}

\subsection{Extração e Síntese dos Dados}

Ao final da seleção, restaram 17 artigos que descrevem jogos nas mais diversas áreas do conhecimento como, por exemplo, Processos de Desenvolvimento de Software, Programação Java, Modelagem Matemática, História, Ciências, Inglês, Ecologia e outros jogos com caráter multidisciplinar. Dentre os estudos avaliados, sete (41\%) referem-se à MMORPG (Massively Multiplayer Online Role Playing Games) [Rezaei e Ghodsi 2014]. Além disso, três jogos [Ye et al. 2007, Shanahan 2009, Emad et al. 2013] foram construídos na plataforma do Second Life [Bardzell e Odom 2008], um conhecido mundo virtual $3 \mathrm{D}$ que permite que os usuários interajam de modo online através de avatares. A maioria dos jogos foi criado por pesquisadores dos Estados Unidos, seguidos por Taiwan e Holanda.

\section{QP 1.1- Quais métodos de avaliação são utilizados?}

$\mathrm{Na}$ maioria dos estudos (13 artigos, 76\%) foram utilizados questionários com o objetivo de avaliar os jogos. Entretanto, somente em dois estudos, os questionários foram apresentados e detalhados pelos autores [Ye et al. 2007, Hoda et al. 2014]. Nestes casos, foram utilizadas perguntas para avaliar a opinião dos jogadores em relação a um conjunto de aspectos. Esses questionários não foram elaborados com o objetivo de serem ferramentas sistemáticas de avaliação, englobando aspectos específicos de jogos multiusuários, como sociabilidade e cooperação. Exemplos de perguntas incluem:

\footnotetext{
"Quais aspectos gerais do Second Life você mais gostou? Por quê?" [Ye et al. 2007]

"Você gostou do jogo?" [Hoda et al. 2014]

"Você gostaria de jogá-lo novamente?" [Hoda et al. 2014]
} 
A observação foi utilizada com o objetivo de avaliar o comportamento dos jogadores em um ambiente multiusuário. Somente quatro estudos não relataram a utilização deste método [Ye et al. 2007, Schmitz et al. 2014, Shanahan 2009, e Natvig et al. 2004]. Como jogos multiusuários geralmente fornecem a possibilidade dos usuários comunicarem entre si através de mensagens digitadas (chats), surgiu a necessidade de gravá-las [Emad et al. 2013, Hauge e Riedel 2012, Admiraal et al. 2011], para posterior análise da interação dos usuários.

Apenas três estudos relatam experimentos utilizando grupos de controle para verificar a eficácia do jogo em relação ao aprendizado [Yoon et al. 2013, Schmitz et al. 2014, Lester et al. 2014]. Oito trabalhos relatam a utilização de uma entrevista para obter feedback imediatamente após o jogo [Hsu et al. 2013, Emad et al. 2013, Schmitz et al. 2014, Hoda et al. 2014, González-González et al. 2014, Lester et al. 2014, Pourabdollahian et al. 2012, Schmitz et al. 2011]. Em seis jogos um grupo de testes foi designado para verificar e validar o jogo antes da disponibilização da versão para o usuário final [Yang e Hsu 2013, Emad et al. 2013, González-González et al. 2014, Lester et al. 2014, Sisler et al. 2008, Natvig et al. 2004]. Finalmente, um artigo [Hauge e Riedel 2012] relata a realização de uma conversa informal para troca de informações entre usuário e avaliador, após a partida.

\section{QP 1.2 - Quais dimensões são avaliadas?}

Identificamos, durante a análise, aspectos que se enquadram nas seguintes dimensões (Figura 1): (i) Interação; (ii) Aprendizado; (iii) Experiência do Usuário (UX); (iv) Envolvimento; (v) Jogabilidade; (vi) Usabilidade; (vii) Desenho da interface; e (viii) Plataforma.

Dos 17 artigos selecionados, $11(65 \%)$ apresentaram uma avaliação de fatores relacionados à interação entre os usuários. Nesses trabalhos, foram considerados os seguintes fatores:

- Sociabilidade: Consiste na habilidade de comunicar e interagir com os outros jogadores, para alcançar algum objetivo, ou satisfazer interesses. Essa é uma característica importante para o trabalho em equipe e foi apontada como uma característica fundamental para o aprendizado. No trabalho de Ye et al. (2007), essa característica foi citada pelos usuários como o aspecto mais importante para $\mathrm{o}$ aprendizado.

- Comunicação: A comunicação pode ser um ponto importante para a execução de tarefas colaborativas em um jogo multiusuário. O trabalho de Hauge e Riedel (2012) faz uma análise dos tipos de comunicação presentes no jogo. Neste trabalho, percebeu-se que os jogadores que desempenhavam um maior número de funções, geralmente comunicavam sobre aspectos mais relevantes para o jogo.

- Competitividade: A competitividade geralmente está associada ao envolvimento do jogador com o jogo. É comum que os usuários tenham mais prazer ao serem desafiados por outros jogadores, como foi descrito no trabalho de Admiraal et al. (2011). Entretanto, em jogos para a educação infantil, como nos trabalhos de Hoda et al. (2014) e González - González et al. (2014), as crianças preferiram colaborar a competir. Isso sugere que para alguns públicos e temas, a competição pode não ser um aspecto a ser destacado. 
- Colaboração: A colaboração é o fator de interação mais aplicado nos jogos. Muitos artigos comprovam que quanto mais os jogadores de um mesmo time ajudam uns aos outros, as tarefas designadas são mais facilmente realizadas. $\mathrm{O}$ trabalho de Hauge e Riedel (2012), por exemplo, faz um paralelo com a vida real, ensinando e comprovando a importância de conceitos do trabalho em equipe.

Somente em dois jogos não foram apresentadas avaliações para verificar o aprendizado [Emad et al. 2013, Hoda et al. 2014]. Os outros 15 (88\%) estudos utilizaram métodos variados para verificar a eficácia educacional dos jogos como, por exemplo, entrevistas e questões a serem respondidas pelos jogadores sobre o aprendizado. Três estudos avaliaram somente este fator [Yang e Hsu 2013, Schmitz et al. 2011, Natvig et al. 2004].

Dados os resultados obtidos, percebe-se que os autores, usualmente, não avaliam a qualidade da interface dos jogos. Somente quatro estudos [Ye et al. 2007, Hsu et al. 2013, Hoda et al. 2014, Lester et al. 2014] discutem esse aspecto. Já o envolvimento dos jogadores foi avaliado em um número considerável de artigos. Muitas vezes os autores ligam esse fator ao desafio proporcionado pelo jogo.

A Figura 1 ilustra a proporção com que cada fator foi avaliado nos 17 estudos primários.

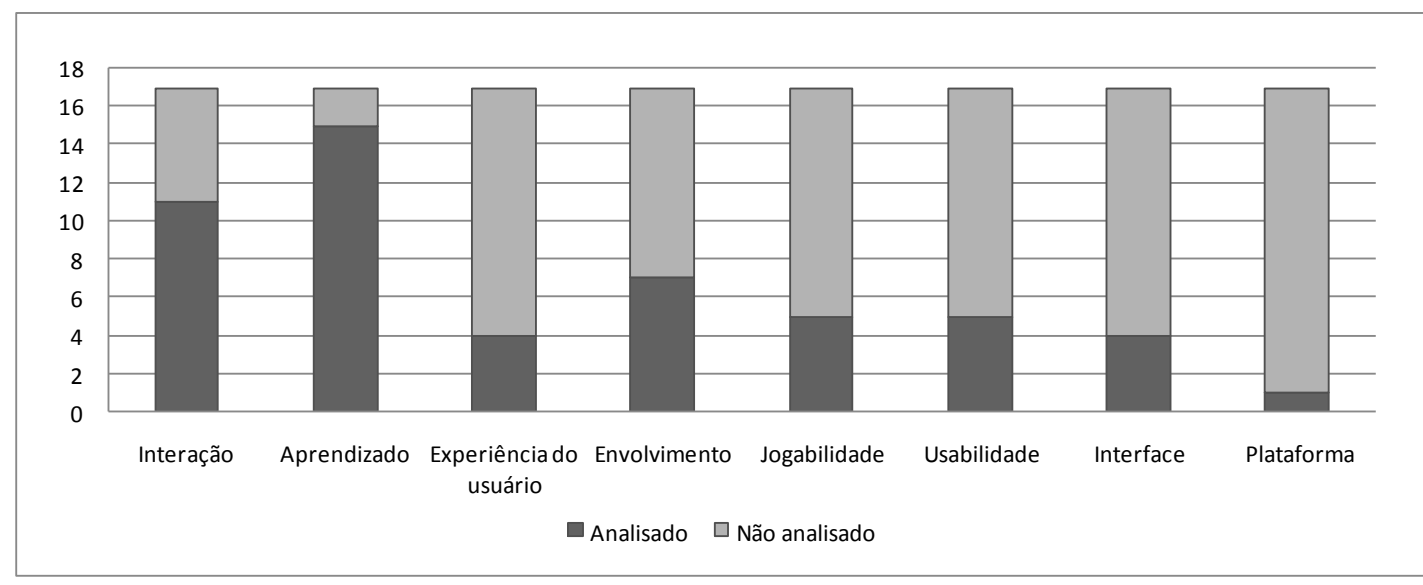

Figura 1. Fatores avaliados nos estudos primários.

\section{QP 1.3- Quão satisfatórios têm sido os resultados das avaliações?}

Os resultados relatados nos estudos mostram um cenário bastante positivo da utilização dos jogos. A maioria dos estudos apresenta dados confirmando a eficácia do jogo no atendimento aos objetivos propostos como, por exemplo, no aprendizado, motivação, e colaboração. Entretanto, observa-se que as avaliações dos jogos educacionais multiusuários estão, principalmente, restritas a testes-piloto de pequeno porte ou avaliações subjetivas. Os artigos não utilizam estudos mais amplos e rigorosos que permitiriam uma verificação mais adequada desses jogos educacionais. Isso é confirmado pela ausência de estudos que realizam, por exemplo, experimento formal para validar a eficácia no aprendizado.

Dado este cenário, percebe-se a necessidade de se criar ferramentas para a avaliação de jogos educacionais multiusuários. A Tabela 3 apresenta uma compilação inicial de um conjunto de questões para avaliação do uso de jogos educacionais 
multiusuários. Essas questões foram identificadas nos estudos primários e em trabalhos relacionados e estão agrupadas com base nas dimensões apresentadas na Figura 1.

Tabela 3 - Questões para avaliação.

\begin{tabular}{|c|c|}
\hline Interação & $\begin{array}{l}\text { 1. Você gostou da oportunidade de interagir com outras pessoas? } \\
\text { 2. Você encontrou dificuldades para resolver os problemas em equipe? } \\
\text { 3. O jogo promove momentos de cooperação entre os participantes? } \\
\text { 4. O jogo promove momentos de competição entre os participantes? }\end{array}$ \\
\hline $\begin{array}{l}\text { Aprendizado } \\
\text { [Savi 2011] }\end{array}$ & $\begin{array}{l}\text { 1. O jogo te ajudou a aprender novos conteúdos? } \\
\text { 2. Foi necessário ter conhecimento prévio para jogar? } \\
\text { 3. O jogo te ajudou a reforçar o conhecimento da disciplina? } \\
\text { 4. Você acha que o jogo é um meio eficiente para o aprendizado do conteúdo, } \\
\text { comparado com outras atividades como leitura, palestra ou pesquisa? }\end{array}$ \\
\hline $\begin{array}{l}\text { Experiência do } \\
\text { Usuário } \\
\text { [Law e Sun 2012] }\end{array}$ & $\begin{array}{l}\text { 1. Este jogo foi adequadamente desafiador para você? Isto é, as tarefas não são } \\
\text { muito fáceis nem muito difíceis. (Desafio) } \\
\text { 2. Você conseguiu atingir os objetivos do jogo por meio das suas habilidades e das } \\
\text { habilidades de seus companheiros? (Competência) } \\
\text { 3. O feedback do jogo te ajudou a entender o que fazer em seguida? (Feedback) }\end{array}$ \\
\hline $\begin{array}{l}\text { Envolvimento } \\
\text { [Keller 2010] }\end{array}$ & $\begin{array}{l}\text { 1. Qual foi o seu grau de atenção no jogo? (Atenção) } \\
\text { 2. O conteúdo do jogo é relevante para os seus interesses? (Relevância) } \\
\text { 3. Ao passar pelas etapas do jogo, você sentiu confiança de que estava } \\
\text { aprendendo? (Confiança) } \\
\text { 4. Você ficou satisfeito com seu desempenho no jogo? (Satisfação) } \\
\text { 5. Você acredita que terá oportunidade de utilizar na prática coisas que você } \\
\text { aprendeu durante o jogo? (Satisfação) }\end{array}$ \\
\hline $\begin{array}{l}\text { Jogabilidade, } \\
\text { Usabilidade e } \\
\text { Interface [Nielsen } \\
\text { 2012] }\end{array}$ & $\begin{array}{l}\text { 1. O jogo é atraente? (Interface) } \\
\text { 2. É fácil utilizar a interface e controle do jogo? (Interface) } \\
\text { 3. As ações disponíveis durante o jogo foram suficientes para alcançar todos os } \\
\text { objetivos? (Jogabilidade) } \\
\text { 4. O funcionamento deste jogo está adequado ao seu jeito de aprender? (Facilidade } \\
\text { de aprendizado) } \\
\text { 5. É fácil aprender como usar os recursos do jogo? (Eficiência) } \\
\text { 6. Você conseguiu fixar as regras do jogo? (Memória) } \\
\text { 7. Os erros cometidos atrapalharam a conclusão do jogo? (Erros) }\end{array}$ \\
\hline
\end{tabular}

\section{Conclusão e Trabalhos Futuros}

Atualmente, existe a necessidade de se ter avaliações mais criteriosas de jogos educacionais multiusuários. Neste trabalho, realizamos um levantamento sistemático da literatura e observamos que as avaliações dos jogos educacionais multiusuários estão, principalmente, restritas a testes-piloto de pequeno porte ou avaliações subjetivas. A partir da revisão, levantamos algumas questões que podem ser analisadas em jogos educacionais multiusuários. Essas questões foram agrupadas nas dimensões descritas na Tabela 3. Como trabalho futuro, iremos melhorar a análise aqui compilada, com o objetivo de criar uma ferramenta de avaliação mais adequada ao contexto de jogos educacionais multiusuários.

\section{Referências - SLR}

Ye, E., Liu, C., Polack-Wahl, J. A. (2007) "Enhancing Software Engineering Education Using Teaching Aids in 3-D Online Virtual Worlds," 37th Frontiers in Education Conference, Milwaukee, USA.

Yang, J. C, Hsu, H. F. (2013) "Effects of prior knowledge on cognitive learning outcomes within an english learning multiplayer online role-playing game," Second IIAI International Conference on Advanced Applied Informatics, p.166-171, Matsue, Japan. 
Hsu, Yu-Jen, Lin, Chang-Hsin, Shih, Ju-Ling (2013) "Developing Multi-player Digital Adventure Education Game with Motion Sensing Technologies," 13th International Conference on Advanced Learning Technologies, p.207-209, Tainan, Taiwan.

Yoon, I., Ng, G., Rodrigues, H., Nguyen, T., Paik, J. H., Yoon, S., Williams, R., Martinez, N. D. (2013) "Iterative Design and Development of the 'World of Balance' Game: From Ecosystem Education to Scientific Discovery," IEEE International Games Innovation Conference, p.283-290, San Francisco, USA.

Emad, S., Broillet, A., Halvorson, W., e Dunwell, N. (2013) "The Competency Building Process of Human Computer Interaction in Game-Based Teaching: Adding the Flexibility of an Asynchronous Format," IEEE International Professional Communication Conference, p.1-8, Vancouver, Canada.

Tobail, A., Crowe, J., Arisha, A., (2011) "Learning by gaming: Supply Chain Application," Proceedings of the 2011 Winter Simulation Conference, p.3935-3946, Ireland.

Schmitz, B., Klemke, R., Specht, M. (2014) "The impact of coupled games on the learning experience of learners at-risk: An empirical study," Pervasive and Mobile Computing, vol.14, p. 57-65.

Hoda, R., Henderson, A., Lee, S., Beh, B., Greenwood, J. (2014) "Aligning technological and pedagogical considerations: Harnessing touch-technology to enhance opportunities for collaborative gameplay and reciprocal teaching in NZ early education," International Journal of Child-Computer Interaction, vol. 2(1), p.48-59.

González-González, C., Toledo-Delgado, P., Collazos-Ordoñez, C., González-Sánchez, J. L. (2014) "Design and analysis of collaborative interactions in social educational videogames," Computers in Human Behavior, vol. 31, February, p. 602-611.

Lester, J. C., Spires, H. A., Nietfeld, J. L., Minogue, J., Mott, B. W., Lobene, E. V. (2014) "Designing game-based learning environments for elementary science education: A narrative-centered learning perspective," Information Sciences, vol. 264, p. 4-18.

Hauge, J. B., Riedel, J. C. K. H. (2012) "Evaluation of simulation games for teaching engineering and manufacturing," Procedia Computer Science, vol. 15, p. 210-220.

Pourabdollahian, B., Taisch, M., Kerga, E. (2012) "Serious Games in Manufacturing Education: Evaluation of Learners' Engagement," Procedia Computer Science, vol. 15, p.256-265.

Admiraal, W., Huizenga, J., Akkerman, S., Dam, G. ten (2011) "The concept of flow in collaborative game-based learning," Computers in Human Behavior, vol.27(3),p. 1185-1194.

Sisler, V., Brom, C., e Slavik, R. (2008) "Towards a Novel Paradigm for Educational Games: The Augmented Learning Environment of 'Europe 2045'," 12th International conference on Entertainment and Media in the Ubiquitous Era, p. 34-38, Tampere, Finland.

Schmitz, B., Czauderna, A., Klemke, R., e Specht, M. (2011) "Game Based Learning for Computer Science Education," Computer Science Education Research Conference, Heerlen, The Netherlands, p. 81-86.

Shanahan, J. (2009) "Students Create Game-Based Online Learning Environment that Teaches Java Programming," 47th Annual Southeast Regional Conference, Clemson, USA.

Natvig, L., Line, S., Djupdal, A. (2004) "'Age of Computers:" An Innovative Combination of History and Computer Game Elements for Teaching Computer Fundamentals," 34th Frontiers In Education Conference, Savannah, USA.

\section{Referências}

Callahan, D., Pedigo, B. (2002) "Educating Experienced IT Professionals by Addressing Industry's Needs," IEEE Software, vol. 19(5), p. 57-62. 
Exter, M. (2014) "Comparing Educational Experiences and On-the-Job Needs of Educational Software Designers," 45th ACM Technical Symposium on Computer Science Education, Atlanta, Georgia, USA, p. 355-360.

Chen, W., Wu, W., Wang, T., Su, C. (2008) "A Game-based Learning System for Software Engineering Education," 38th Frontiers in Education Conference, New York, USA.

Martin, S., Diaz, G., Sancristobal, E., Gil, R., Castro, M., Peire, J. (2011) "New technology trends in education: Seven years of forecasts and convergence," Computers \& Education, vol. 57(3), November, p. 1893-1906.

Navarro, E. O. (2006) "SimSE: A Software Engineering Simulation Environment for Software Process Education," PhD thesis, Donald Bren School of Information and Computer Sciences, University of California, Irvine.

Peixoto, Daniela C. C., Resende, R. F., Pádua, C. I. P. S. (2014) "Evaluating Software Engineering Simulation Games: The UGALCO framework," 44th Frontiers in Education Conference, Madrid, Spain.

Garris, R., Ahlers, R. and Driskel J. E. (2002) "Games, Motivation, and Learning: A Research and Practice Model," Simulation \& Game, vol. 33(4), p. 441-467.

Pietruchinski, M. H., Neto, J. C, Malucelli, A., Reinehr, S. (2011) "Os jogos educativos no contexto do SBIE: uma revisão sistemática de literatura," Anais do XXII Simpósio Brasileiro de Informática na Educação, Aracaju, Brasil.

De Grove, F., Bourgonjon, J., Looy, J. V. (2012) "Digital games in the classroom? A contextual approach to teachers' adoption intention of digital games in formal education," Computers in Human Behavior, vol. 28(6), p. 2023-2033.

Hays, R. T. (2005) "The effectiveness of instructional games: A literature review and discussion," Naval Air Warfare Center Training Systems Division, ACM International Conference Proceeding Series, p. 9-15, Orlando, USA.

Mayer, I. (2012) "Towards a Comprehensive Methodology for the Research and Evaluation of Serious Games," Procedia Computer Science, vol.15, 2012, p. 233-247.

Villalta, M., Gajardo, I., Nussbaum, M., Andreu, J. J., Echeverría , A., e Plass, J. L. (2011) "Design guidelines for Classroom Multiplayer Presential Games (CMPG)," Computers \& Education, vol. 57(3), November, p. 2039-2053.

Keller, J. M. (2010) "Motivational Design for Learning and Performance: The ARCS model approach," Springer, Science + Business Media, LLC.

Savi, R. (2011) "Avaliação de jogos voltados para a disseminação do conhecimento," Universidade Federal de Santa Catarina, Tese de Doutorado, Florianópolis.

Kitchenham, B., e Charters, S. (2007) "Guidelines for Performing Systematic Literature Reviews in Software Engineering," EBSE TR-2007-01, Departments of Computer Science, Keele University and University of Durham, UK.

Rezaei, S., Ghodsi, S. S. (2014) "Does value matters in playing online game? An empirical study among massively multiplayer online role-playing games (MMORPGs)," Computers in Human Behavior, vol. 35, p. 252-266.

Bardzell, S., e Odom, W. (2008) "The Experience of Embodied Space in Virtual Worlds: An Ethnography of a Second Life Community Space and Culture," Space and Culture, vol. 11(3) p. 239-259.

Law, E. L., Sun, X. (2012) "Evaluating user experience of adaptive digital educational games with Activity Theory," Int. Journal of Human-Computer Studies, vol.70 (7), p. 478-497.

Nielsen, J (2012) “Usability 101: Introduction to Usability," Nielsen Norman Group, disponível em: < http://www.nngroup.com/articles/ >, acessado em: 24 fev. 2015. 